\title{
A Study on the Shielding Ability of Current Aerospace Materials against High Energy Cosmic Rays
}

\author{
LIU Dong ${ }^{1}$ \\ BK21plus Clean Energy Convergence and Integration Center for Human Resources Training and \\ Education \\ Jeju National University, Jeju 63243, South Korea \\ E-mail: LIUDONGCN@jejunu.ac.kr

\section{Jong-Kwan Woo} \\ Department of Physics, Jeju National University \\ Jeju National University, Jeju 63243, South Korea \\ E-mail:w00jk@jejunu.ac.kr
}

\begin{abstract}
High energy cosmic rays are one of the biggest concerns for a detector used in space and manned spaceflight, along with the swift development of the high energy experiments in space and spaceflight enterprise, the research of shielding materials against high energy cosmic rays has become increasingly important. In this study, firstly we defined a simple detector in the form of human body, some shielding materials and the geometry of shielding structure, then we comprehensively considers the radiation shielding properties of various materials together with the consideration of mass problems of shielding materials. The radiological protection capability of materials against certain higher energy cosmic ray was appraised from the perspectives of radiation dose. The results concluded under the same level of mass of shielding materials, it is advantageous to employ the composites and non-metal materials as shielding materials than using metal or alloy. For practical application, the cabin wall of spaceship is usually consisted with multi-layers. The calculation result shows that a multi-layers cabin wall that has low density layer-high density layer-low density layers structure has a more ideal shielding effect.
\end{abstract}

\section{Introduction}

For spaceflight missions, one of the main concerns is space radiation. Therefore, the protection against space radiation is a necessity, and the shielding capability of aerospace materials is an important concern for the construction of spacecrafts. The main purpose of this study is to evaluate the shielding effects of several current aerospace materials. This is significant for future spaceflight missions.

XXXIX International Conference on High Energy Physics

4-11 July 2018

Seoul, Korea

\footnotetext{
${ }^{1}$ Speaker

(c) Copyright owned by the author(s) under the terms of the Creative Commons Attribution-NonCommercial-NoDerivatives 4.0 International License (CC BY-NC-ND 4.0).
} 


\section{Space Radiation Environments and Current Astronautic Structural Materials} Considering the features of current and near future manned spaceflights, in this study, the major space radiation sources are assigned as Solar Cosmic Rays (SRCs) and Galactic Cosmic Rays (GCRs). [1,2 and 3] In this study, the current astronautic materials are divided into metallic materials, composites and non-metallic materials and special materials [4 and 5].

\section{Simulated Calculation}

The radiation dose in human phantom under the exposure of SCRs and GCRs are calculated. The illustration of simulation is shown in Fig.1, and the picture on right side shows the crosssection of the cabin.

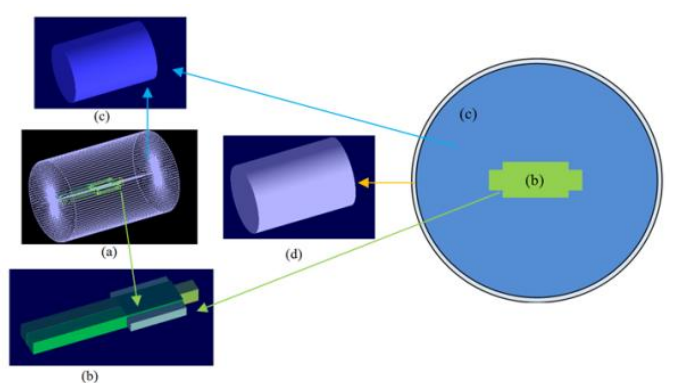

Fig.1. The illustration of the simulation 1. (a) is a general view, (b) is the phantom located at the center of the cabin of the spaceship, (c) is the life environment inside the cabin for astronauts, set with air. The (d) shows the cabin wall structure.

\section{Result and Conclusion}

1. The shielding ability of composites and non-metal materials are better than metal materials and alloys.

2. For metal alloys, the shielding performance of aluminum alloys is similar to that of magnesium alloys. The shielding performance of titanium alloys is lower than that of aluminum and magnesium alloys. The shielding effect of steel is the lowest for all selected metal alloys.

3. For fiber-reinforced composites, polyethylene and carbon based fiber-reinforced composites are better than metal-matrix based composites.

To sum up, as for shielding materials, the shielding effects decreased with the increase of mean atomic number. Therefore, low atomic number materials should be used as much as possible. Among light materials, the support materials, such as liquid hydrogen and water have the best shielding effects.

\section{Reference}

[1] Managing Space Radiation Risk in the New Era of Space Exploration. The National Academic, Washington, D.C., 2008, p.27

[2] Managing Space Radiation Risk in the New Era of Space Exploration. The National Academic, Washington, D.C.,2008, p.28

[3] Information Needed to Make Radiation Protection Recommendations for Space Missions Beyond Low-Earth Orbit. NCRP Report No.153, 2006, p.41

[4] Adrian P Mouritz. Introduction to Aerospace Materials. Woodhead Publishing Limited 2012

[5] Prasad, N. Eswara, Wanhill, R. J. H. Aerospace Materials and Material Technologies Volume 1: Aerospace Materials. 2016 